Revista

\title{
Multi-Ensayos
}

Vol. 6, núm. 12

ISSN: 2412-3285

https://multiensayos.unan.edu.ni

DOI: https://doi.org/10.5377/multiensayos.v6i12.10119

\section{La lucha por la paz y el progreso con justicia social: Compromisos revolucionarios promovidos por el Gobierno de Reconciliación y Unidad Nacional en Nicaragua}

\section{The fight for peace and progress with social justice: Revolutionary commitments promoted by the Government of Reconciliation and National Unity in Nicaragua}

Junieth Osegueda Herrera ${ }^{1}$

Recibido: 14 de enero de 2020. Aceptado: 14 de julio de 2020

"El Frente Sandinista, a la cabeza de las masas populares de Nicaragua, se sacrifica no para alcanzar una mezquina migaja para el pueblo, sino para lograr una radical transformación social y nacional". Carlos

Fonseca Amador

"The Sandinista Front, at the head of the popular masses of Nicaragua, sacrifices itself not to achieve a petty crumb for the people, but to achieve a radical social and national transformation". Carlos Fonseca

Amador

\section{RESUMEN}

El presente ensayo es personal argumentativo y tiene por objetivo exponer los cambios más significativos en cuanto a la lucha por la paz y el progreso con justicia social en Nicaragua a partir del año 2007 con la administración del Gobierno de Reconciliación y Unidad Nacional (GRUN), liderado por el Comandante Daniel Ortega Saavedra y la compañera Rosario Murillo Zambrana. Es decir, la transición de un período de 16 años con gobiernos serviles a los intereses del imperio estadounidense, quienes promovieron políticas neoliberales que sumergieron al País en el atraso, la dependencia económica y la desigualdad social, a un período de lucha por la paz y el progreso con justicia social promovido por el GRUN por medio de la ejecución de Políticas y Proyectos Sociales y Públicos, con alianzas de cooperación y acuerdos comerciales con otros Países, y con la promulgación de la Ley N 985 (Ley para una cultura de diálogo, reconciliación, seguridad, trabajo y paz).

Palabras clave: paz; progreso con justicia social; revolución; políticas y proyectos sociales.

1 Docente del Departamento de Ciencias Económicas y Administrativas de la Facultad Regional Multidisciplinaria de Estelí. juniethosegueda@gmail.com

(c) 2020 Revista Multi-Ensayos.

(c) (i) (-) Este trabajo está licenciado bajo una Licencia Internacional Creative Commons 4.0 Atribución-NoComercial-Compartirlgual. 


\section{ABSTRACT}

This essay is argumentative and its objective is to expose the most significant changes in the fight for peace and progress with social justice in Nicaragua since 2007 with the administration of the Government of Reconciliation and National Unity (GRUN), led by Commander Daniel Ortega Saavedra and comrade Rosario Murillo Zambrana. In other words, the transition from a period of 16 years with servile governments to the interests of the American empire, who promoted neoliberal policies that plunged the country into backwardness, economic dependency, and social inequality, to a period of struggle for peace and the progress with social justice promoted by the GRUN through the execution of Social and Public Policies and Projects, with cooperative alliances and commercial agreements with other Countries and with the promulgation of Law No. 985 (Law for a culture of dialogue, reconciliation, security, work and peace).

Keywords: peace; progress with social justice; revolution; social policies and projects.

\section{INTRODUCCIÓN}

Desde el año 1990 hasta el 2006, los gobiernos de derecha ejecutaron en nuestro país, políticas neoliberales diseñadas por el Fondo Monetario Internacional (FMI) y el Banco Mundial (BM), ambos organismos subyugados por el imperio estadounidense. Generando en consecuencia; descontento social por el irrespeto a los derechos fundamentales de los y las nicaragüenses, por el atraso, la inseguridad ciudadana y jurídica, la desigualdad social y la dependencia económica en la que sumergieron al país durante ese período.

Con la administración y gestión del GRUN a partir del año 2007, Nicaragua se ha redirigido hacia la lucha por el mantenimiento de la paz y el progreso con justicia social para la restitución y protección de derechos por medio de políticas y proyectos públicos con alianzas de cooperación y acuerdos comerciales con otros países.

Es decir, hacia la continuidad de la Revolución popular sandinista de 1979, en honor a nuestros héroes y mártires de todas las generaciones: Benjamín Zeledón, el héroe anti-ntervencionista, Augusto C. Sandino, padre de la Revolución popular antimperialista, Rigoberto López Pérez, héroe iniciador del principio del fin de la dictadura Somocista, Carlos Fonseca Amador, el más alto continuador de la gesta heroica del general de hombres y mujeres libres, fundador del Frente Sandinista de Liberación Nacional y jefe de la Revolución popular sandinista. Héroes que ofrendaron sus vidas frente a la agresión imperialista, forjaron y desarrollaron la lucha por una Nicaragua libre, soberana e independiente para garantizar la paz y la felicidad a las futuras generaciones.

\section{DESARROLLO}

A cuarenta y un años, la Revolución popular sandinista continúa más firme que nunca, resistiendo las agresiones imperialistas y avanzando hacia el fortalecimiento de la paz y el progreso con justicia social. Avances promovidos por el Frente Sandinista de liberación Nacional desde la administración y gestión del GRUN, enfrentando y venciendo a los enemigos de la humanidad, como tantas veces lo ha hecho el 
pueblo nicaragüense, constituyéndose así en una patria digna, valiente, soberana, libre y ejemplar para todos nuestros pueblos latinoamericanos que históricamente han sido agredidos por los Estados Unidos de Norte América.

Así pues, el Gobierno de Reconciliación y Unidad Nacional puso en marcha a partir de 2007 su estrategia de crecimiento económico y lucha Contra la Pobreza, cuyo perfil de mediano plazo está contenido en el Plan Nacional de Desarrollo Humano (PNDH), dado a conocer oficialmente en abril de 2008 en forma preliminar y en forma definitiva en octubre de 2008. Este Plan fue estructurado como cambio de modelo neoliberal que está en crisis mundialmente hacia el modelo del poder ciudadano, descrita en el capítulo 4. El modelo neoliberal está en crisis porque había puesto al mercado como el centro del modelo, capaz de auto regularse, pero la crisis económica internacional ha demostrado que el mercado es imperfecto, que requiere instrumentos de regulación, y además puede producir resultados que pueden ser socialmente no deseables en términos de desigualdad, lo cual a su vez requiere la intervención del Estado para que sean corregidos. (GRUN, Plan nacional de desarrollo humano actualizado 2009-2011, 2009, pág. 7)

En otras palabras, el PNDH es un plan macro para la planificación institucional de políticas públicas a mediano y largo plazo. Su estructura es holística ya que abarca de forma integral todos los lineamientos que responden a las demandas sociales de las y los nicaragüenses. En este sentido el GRUN está luchando por establecer por medio de este plan las condiciones que propicien la generación de trabajo digno y superación de la pobreza, la desigualdad social, así como la eliminación del hambre. De igual manera, promueve el uso adecuado y racional de los recursos naturales, y la protección al medio ambiente para garantizar la vida y la supervivencia.

Conviene subrayar, que el PNDH se sustenta con los ejes: desarrollo social, educación técnica tecnologías y conocimiento, equidad de género, Comunidades Indígenas y Afrodescendientes, trabajo y prosperidad, desarrollo socio productivo, política monetaria y financiera. Así como políticas fiscales y aduaneras, políticas ambientales y de protección de los recursos naturales, infraestructuras, viviendas familiares, promoción y facilitación de las inversiones. Además, el desarrollo local, desarrollo urbano, gestión de riesgo frente a desastres y calamidades, seguridad soberana, cambio climático y financiamiento, en procura de garantizar el desarrollo humano sostenible de la nación. (GRUN, Ejes del programa nacional de desarrollo humano, 2017).

En efecto, dichos ejes se han adoptado en consonancia con los diecisiete objetivos de desarrollo sostenible de la Agenda 2030 de la organización de naciones unidas (ONU). Objetivos con los cuales, la sociedad internacional, principalmente los países más industrializados, deben asumir con responsabilidad su compromiso y solidaridad con los pueblos más vulnerables del mundo, para alcanzar el verdadero desarrollo humano sostenible con respeto a la independencia, soberanía y autodeterminación nacional de los mismos, con justicia social, igualdad, equidad de género, políticas ambientales que protejan al planeta, garantizando la vida y sobrevivencia humana, la protección a la madre tierra y la paz y seguridad internacional. 
Por tanto, el artículo 5 de la Constitución política de Nicaragua mandata que: "Nicaragua fundamenta sus relaciones internacionales en el principio del no alineamiento, en la búsqueda de la paz, y en el respeto a la soberanía de todas las naciones; por esto, se opone a cualquier forma de discriminación, es anticolonialista, antirracista, y rechaza toda subordinación de un Estado a otro Estado". (Asamblea, 1987).

En palabras del canciller de Nicaragua Denis Moncada Colindres: continuaremos promoviendo las relaciones internacionales de Nicaragua con todos los Pueblos y Gobiernos del mundo, a nivel bilateral, regional y multilateral, en el marco de la Constitución Política y el Derecho Internacional, sustentados en la 6 Vocación de Paz y en los principios de libertad, Independencia, autodeterminación, seguridad soberana, no agresión y solidaridad. (MINREX, 2020, págs. 5-6).

Con respecto a los cambios significativos relacionados con la lucha contra el narcotráfico y crimen organizado para garantizar la paz y la seguridad en el país, la Asamblea Nacional aprobó la Ley de Reformas y Adiciones a la Ley contra el Lavado de Activos, el Financiamiento al Terrorismo y el Financiamiento a la Proliferación de Armas de Destrucción Masiva. Ley que busca fortalecer la estrategia del muro de contención contra el crimen organizado y narcotráfico internacional que el GRUN ha venido ejecutando desde el año 2007. Esta reforma tiene como objetivos fundamentales reafirmar el compromiso que tiene el estado de Nicaragua en la lucha contra la delincuencia organizada y su financiamiento a través del fortalecimiento constante de nuestro marco jurídico.

La iniciativa establece la ampliación del listado de actividades profesionales no financieras relevantes, entre estos los abogados y notarios públicos, "para que apliquen medidas preventivas con enfoque basado en los riesgos, y que designe al Poder Judicial como su supervisor en materia antilavado de activos, contra el financiamiento al terrorismo y contra el financiamiento a la proliferación de armas de destrucción masiva". (19digital, Asamblea Nacional aprueba reforma a ley 977 para fortalecer lucha contra el terrorismo, crimen organizado y lavado de dinero, 2019).

Estos avances relacionados con la lucha contra el narcotráfico y el crimen organizado se han logrado gracias a las labores arduas tanto del Ejército, como de nuestra gloriosa Policía Nacional de Nicaragua, cuyos miembros se han mostrado leales a los ideales revolucionarios. Razón por la cual las y los nicaragüenses debemos ser conscientes de apoyarles, asumiendo nuestro rol como ciudadanos responsables de contribuir a generar cambios sociales que aseguren nuestro bien común.

Por lo que se refiere a proyectos de infraestructura vial, a continuación, se detallan los cinco proyectos más significativos, ejecutados por el GRUN el pasado año 2019:

1. El 21 de marzo del 2019, el Paso a Desnivel del 7 Sur fue inaugurado por el Presidente Daniel Ortega, la Compañera Rosario Murillo y las familias nicaragüenses. para mejorar la circulación vial en la capital. Es un punto en el que se concentran 5 de las carreteras más importantes de Managua que son: la Carretera Vieja León, la Carretera Nueva a León, la Carretera Sur, el segmento hacia el Norte y hacia el Sur y la pista Juan Pablo II, y tiene la característica de que cuenta con una rotonda en la parte baja que se integra a la nueva Pista Juan Pablo II.

2. Paso a desnivel Nejapa, una de las obras viales más importantes en Managua. Antes de la construcción 
del proyecto del Paso a desnivel 7 sur y el Paso desnivel Las Piedrecitas, para poder entrar a la ciudad de Managua los conductores tenían que esperar al menos 45 minutos y hoy en menos de 1 minuto los vehículos pueden pasar por la nueva intersección. En la intersección del 7 sur, los conductores esperaban 30 minutos y ahora el tiempo que toma recorrer ese tramo es de 2 a 3 minutos.

3. El 30 de abril del 2019, durante el acto central en conmemoración del séptimo aniversario del paso a otro plano de vida del fundador del FSLN, Comandante Tomás Borge Martínez, el Presidente de Nicaragua, Comandante Daniel Ortega inauguró oficialmente la carretera de concreto hidráulico de 72.8 kilómetros que une por primera vez en la historia de Nicaragua el Pacífico y la región del Caribe nicaragüense.

4. Después de 500 años, por primera vez en la historia de Nicaragua se hizo realidad el proyecto de construcción de la carretera de Bluefields, la cual tiene 170 mil metros cúbicos de concreto hidráulico, que garantizan la durabilidad de la obra en el mediano y largo plazo. En este proyecto participaron más de 900 obreros que trabajaron día y noche para finalizar este proyecto en el tiempo estipulado y el costo total de la obra fue aproximadamente de 115 millones de dólares. La obra tiene 50 años de vida útil y cuenta con la señalización adecuada, garantizando así una circulación correcta para las familias de la costa Caribe.

5. Inauguración del nuevo tramo carretero Río BlancoMulukukú, Siuna. En esta nueva ruta se movilizan más de 19 mil 200 pasajeros por mes, y desde Managua se movilizan al menos 24 mil pasajeros por mes. (19digital, 5 proyectos del 2019 marcados en la historia de Nicaragua, 2019).

Por otro lado, es importante destacar los proyectos de electrificación gestionados y ejecutados por el GRUN. En los cuales ha invertido más de 3 mil millones de córdobas, proyectos ejecutados durante el año 2019. En cuyo caso se sobrepasó la meta de electrificación establecida del $97 \%$ de cobertura eléctrica a nivel nacional. "Cumplimos y eso es lo que nos satisface a los funcionarios y a nuestro Gobierno, sentir que le cumplimos a nuestro pueblo, pero también que sobrepasamos nuestra meta", (19digital, Nicaragua sobrepasa la meta de electrificación para el 2019, 2019).

En cuanto a los cambios significativos en el sector educación, se ha avanzado en alcanzar calidad, equidad e inclusión educativa en nuestro país, destacando:

- El proceso de Formación de docentes en todos los niveles y modalidades.

- La incorporación del inglés como segundo idioma en educación primaria pública.

- La ampliación de Tecnologías Educativas y la promoción del uso con fines educativos.

- El Trabajo conjunto de los subsistemas educativos, CNU-INATEC-MINED.

- El Programa de Nutrición Escolar.

- La dignificación de ambientes escolares.

- La Promoción de valores y el cuido de la vida.

- Fortalecimiento de la educación artística y cultural como parte de la formación integral de

Estudiantes. (19digital, Ministra de Educación destaca logros en el cumplimiento de las metas de los Objetivos de Desarrollo Sostenible, 2019). 
Esos esfuerzos del GRUN por medio del Ministerio de educación, Cultura y Deportes, han garantizado que a partir del año 2007 la educación en Nicaragua sea gratuita, igualitaria, inclusiva, con calidad y pertinencia. Es decir, integral. No hay que olvidar que la educación es el pilar fundamental para la transformación de las y los nicaragüenses y para lograr cambios sociales significativos.

Otro cambio social significativo promovido por el GRUN desde el año 2007 ha sido la construcción y equipamiento de 18 nuevos hospitales, actualmente hay 6 en construcción y 8 más que se construirán próximamente.

Hospitales primarios construidos:

1. Hospital Primario San Juan de Río Coco - Madriz.

2. Hospital Primario Muelle de los Bueyes - Chontales.

3. Hospital Primario Héroes y Mártires de San José de las Mulas, La Dalia, Matagalpa.

4. Hospital Primario Carlos Fonseca - Mulukukú - RACCN.

5. Hospital Primario Prinzu Pawanka, Prinzapolka - RACCN.

6. Hospital Primario El Sauce.

7. Hospital Primario de Chichigalpa.

8. Hospital Primario de San Francisco Libre, Managua.

9. Hospital primario en El Jícaro.

10. Hospital primario en Corn Island.

11. Hospital Primario de Tipitapa.

12. Hospital Primario en San José de Bocay.

13. Hospital Primario de la CMP Jinotega.

14. Hospital Primario de San Juan del Sur.

15. Hospital Primario de San Miguelito, Río San Juan.

Hospitales nacionales construidos:

16. Hospital Solidaridad, Managua.

17. Hospital Fernando Vélez Páiz, Managua

18. Hospital departamental:

19. Hospital departamental de Boaco.

\section{Actualmente en construcción: 6}

1. Hospital Primario San José, Matiguás, Matagalpa.

2. Hospital Departamental de Chinandega.

3. Hospital Departamental de Nueva Segovia.

4. Hospital Oscar Danilo Rosales de León.

5. Hospital Regional Nuevo Amanecer de la Región Autónoma de la Costa Caribe Norte.

6. Hospital Primario Los Chiles, Río San Juan.

Hospitales que se van a construir próximamente: 8

1. Hospital Departamental de Nueva Guinea, Zelaya Central.

2. Hospital Primario Bello Amanecer, Quilalí, Nueva Segovia. 
3. Hospital Pastor Jiménez, Jalapa, Nueva Segovia.

4. Hospital Primario Fidel Ventura, Waslala, Matagalpa.

5. Hospital Primario Jorge Navarro, Wiwilí, Jinotega.

6. Hospital Primario Mina El Limón, Larreynaga, León.

7. Hospital departamental de Siuna.

8. Hospital Aldo Chavarría, Managua. (19digital, Gobierno Sandinista ha construido 18 hospitales desde el 2007, 2020).

Cabe recalcar que nuestro modelo de salud impulsado por el Comandante Daniel Ortega y la compañera Rosario Murillo está basado en restituir ese derecho gratuito y accesible en igualdad de condiciones, promover la responsabilidad compartida entre las y los nicaragüenses y el Estado. Ante todo, con el protagonismo del individuo, las familias y la comunidad en general. Lo cual es posible con el acompañamiento de los gobiernos locales, instituciones públicas en coordinación con el Ministerio de Salud (MINSA). Para lo cual el GRUN continua ejecutando acciones que apuntan al fortalecimiento del Sistema Nacional de Salud, en especial su capacidad de respuesta sobre todo ante epidemias, ante pandemias como el Covid-19, en procura de reducir los riesgos y el impacto de las mismas en la población nicaragüense.

Es preciso resaltar también, que desde el año 2007 el GRUN ha entregado más de 49 mil soluciones de viviendas a igual número de familias, quienes hoy están contentas porque su derecho a una casa digna fue restituido por la administración y gestión de nuestro bueno gobierno.

Un aspecto clave ha sido el programa de viviendas Bismark Martinez, el cual se creó como parte de la restitución del derecho a una vivienda digna, lo cual ha significado un sueño hecho realidad para las y los nicaragüenses más desfavorecidos. "A nivel nacional son cincuenta mil viviendas las que se entregarán en los próximos años. Solo en Managua se entregarán 20 mil viviendas". (19digital, 2020). Esta transformación de vida solo ha sido posible por la voluntad política del presidente Daniel Ortega y la vicepresidenta Rosario Murillo, que, conscientes de la necesidad de muchos nicaragüenses a una vivienda digna, decidieron ejecutar este maravilloso proyecto de viviendas a bajo costo para dignificar a las familias nicaragüenses.

Asimismo, el GRUN también ha creado el Plan de políticas y proyectos de desarrollo de: energía, transporte, agua y saneamiento, puertos, aeropuertos, telecomunicaciones, turismo y pesca para potenciar la inversión e incrementar la competitividad del país y elevar los niveles de cobertura y calidad de los servicios públicos durante el período: 2019-2021. El fin es dinamizar el crecimiento económico del país, impulsando el comercio, la movilización de bienes, mercancías y personas y el acceso a nuevos centros de producción. Este plan constituye una herramienta para la negociación de recursos financieros provenientes de gobiernos y organismos financieros internacionales y de inversionistas tanto nacionales como extranjeros, lo cual genera fuentes de empleo y prosperidad para las familias nicaragüenses.

En efecto, con el referido plan el GRUN promueve la búsqueda de nuevos mercados y la celebración de nuevos acuerdos comerciales para la colocación de nuestros productos nacionales de la oferta exportable real y potencial; Cuyas políticas previstas para la búsqueda de nuevos mercados son las siguientes: 
- Aprovechar acuerdos comerciales existentes: CAFTA-DR (Tratado de libre comercio entre Estados Unidos, Centro América y República Dominicana), Unión Europea, Taiwán, Panamá, Venezuela, Cuba, Ecuador, México, Chile y Corea del Sur.

- Finalizar firma de acuerdos comerciales con: ALADI (Asociación latinoamericana de integración) y Reino Unido.

- Promover la firma de nuevos acuerdos comerciales con: Japón, Turquía, India y Unión Aduanera Euroasiática.

- Desarrollar la promoción de relaciones comerciales con los mercados de República Popular China y países árabes.

- Consolidar y fortalecer la integración económica centroamericana, Zona de Libre Comercio, Unión Aduanera y Mercado Común, promoviendo la eliminación de obstáculos al Libre Comercio, la armonización de las normas sanitarias y fitosanitarias; la congruencia de las políticas nacionales de transporte; y la coordinación política productivas. (PRONicaragua, 2019)

Igualmente, por medio de las referidas Políticas y Proyectos públicos, el GRUN promueve: La aplicación de la estrategia Centroamericana de facilitación del comercio y competitividad con énfasis en la gestión coordinada de fronteras; y la política centroamericana de movilidad y Transporte; ambas aprobadas en la reunión de presidentes de Centroamérica.

En relación a la lucha por la paz, en el año 2018 las y los nicaragüenses enfrentamos el fallido golpe de Estado impulsado por la fracasada oposición de Nicaragua, quienes promovieron delitos contra la vida y la seguridad personal; asesinatos y secuestros. Además, delitos contra la tranquilidad pública; terrorismo y crimen organizado, delitos contra la libertad e integridad sexual; violaciones y abusos sexuales, que provocaron daños psicológicos y hasta muerte a personas nacionales y extranjeras. Así como la destrucción de la propiedad pública y privada en nuestro país.

Es preciso insistir en que dichos acontecimientos fortalecieron la fe en nuestro señor Jesucristo, el amor patriótico, el amor al prójimo, la empatía, la solidaridad, la tenacidad, el compromiso social y la unidad del pueblo sandinista para continuar apoyando al GRUN en este proceso de sanación espiritual, reconciliación y recuperación del crecimiento económico que habíamos alcanzado hasta antes del 18 de abril del 2018.

En palabras del comandante Daniel Ortega: "Hermanos nicaragüenses, familias nicaragüense, somos un pueblo con mucha fe, con mucha tenacidad, un pueblo que es laborioso, creativo; todas esas virtudes que tiene la especie humana, las tiene también el pueblo nicaragüense y esas virtudes, acompañadas de valor de la solidaridad, se convierte en una potencia en momentos como este, para retomar o abrir nuevos caminos, nuevas rutas, para la paz, la estabilidad y el bienestar de las familias nicaragüenses, por eso no nos cansaremos de repetir que ¡Nicaragua quiere Trabajo, Paz y Reconciliación!. (19digital, Comandante Daniel Ortega: La lucha por la paz, sigue siendo un reto y un objetivo a alcanzar en el mundo, 2018).

En consecuencia, el GRUN ha promulgado y puesto en vigencia por medio de la Asamblea Nacional de Nicaragua, la Ley $N^{\circ} 985$ (Ley para una cultura de diálogo, reconciliación, seguridad, trabajo y paz), en virtud de la cual se crearon e instalaron más de 7000 comisiones de reconciliación, justicia y paz en todo el territorio nacional. Comisiones compuestas por líderes comunitarios; religiosos, enfermeras, parteras, 
atletas, artistas, maestros, entre otros, que se han destacado en cada comunidad. En este caso, han asumido la responsabilidad de promover y defender una cultura de vida con fraternidad, que propicie la convivencia pacífica, la concordia, la hermandad y la solución de diferencias entre nicaragüenses por medio del diálogo.

De igual manera, las referidas comisiones brindan acompañamiento solidario en los programas sociales del plan nacional de desarrollo humano para contribuir al progreso con justicia social, restituyendo derechos en respuesta a las justas exigencias del bien común de las y los nicaragüenses.

Concretamente, el artículo 2 de la referida Ley 985 establece que el objetivo de la misma es contribuir al fortalecimiento de un Estado Democrático y Social de Derecho, que promueva una Cultura de Diálogo, Reconciliación, Seguridad, Trabajo y Paz, en aras de salvaguardar la paz, la estabilidad, el bien común y la convivencia pacífica entre las y los nicaragüenses, fundamentado en el respeto a la vida, la libertad, la justicia, la igualdad, la equidad, la solidaridad, la inclusividad, la interculturalidad, el respeto a la Madre Tierra y los Derechos Humanos, tomando en cuenta el legado Cultural de las Familias nicaragüenses en la creación y fortalecimiento de una Cultura de Diálogo, Reconciliación, Seguridad, Trabajo y Paz que permita incidir en la prevención de todas las expresiones de desencuentro entre las Personas, las Familias y las Comunidades.. (Asamblea, Ley para una cultura de diálogo, reconciliación, seguridad, trabajo y paz, 2019, pág. 4).

En suma, por medio de esta Ley el GRUN promueve valores tales como la tolerancia, la empatía, la solidaridad, la hermandad, el respeto a la identidad cultural, así como los derechos y deberes de las y los nicaragüenses en el proceso de socialización. Es decir que promueve la adopción de actitudes y comportamientos orientados a erradicar la violencia y propiciar la sana convivencia que favorezca el desarrollo humano sostenible en nuestro país.

\section{CONCLUSIONES}

Para concluir, es meritorio reconocer la excelente administración y gestión del Gobierno de Reconciliación y Unidad Nacional, liderado por el Comandante Daniel Ortega Saavedra y por la compañera Rosario Murillo Zambrana. Sobre todo, en su lucha constante por suprimir el atraso y la dependencia heredados por los anteriores gobiernos neoliberales, para garantizar la paz y el progreso con justicia social a la población nicaragüense, por medio de la ejecución de políticas y proyectos sociales y públicos recogidos en el PNDH, los cuales han respondido a las justas exigencias del bien común.

Ante todo, el PNDH está en constante construcción en pro de garantizar tanto el crecimiento económico del país, como el bienestar social, siendo este último un imperativo para mejorar la calidad de vida de las y los nicaragüenses. Es decir, la realización plena de las y los nicaragüenses, que es lo que caracteriza a nuestro modelo de desarrollo del poder ciudadano, el cual apuesta al desarrollo humano sostenible.

No hay que olvidar el esfuerzo y productividad del pueblo sandinista, pueblo heroico, laborioso, cristiano y solidario. Compuesto hoy, por: madres de héroes y mártires, juventud gloriosa, niños y niñas que anhelan crecer en un ambiente de paz y seguridad, hombres y mujeres libres: obreros, campesinos y 
profesionales quienes con su trabajo digno contribuyen cada día con la defensa de la economía nacional y con el fortalecimiento de la paz y la seguridad en toda la nación.

Igualmente, se reconoce el aprovechamiento de los acuerdos comerciales existentes: CAFTA-DR (Tratado de libre comercio entre Estados Unidos, Centro América y República Dominicana), Unión Europea, Taiwán, Panamá, Venezuela, Cuba, Ecuador, México, Chile y Corea del Sur, concluir firma de acuerdos comerciales con: ALADI (Asociación latinoamericana de integración) y Reino Unido. Igualmente, el GRUN promueve la firma de nuevos acuerdos comerciales con: Japón, Turquía, India y Unión Aduanera Euroasiática. Además, desarrolla relaciones comerciales con los mercados de República Popular China y países árabes para poder alcanzar el crecimiento económico y el desarrollo humano sostenible en la nación.

Por último, es menester reconocer la importancia de la promulgación y entrada en vigencia de la Ley $N^{\circ}$ 985 (Ley para una cultura de diálogo, reconciliación, seguridad, trabajo y paz), que fue creada por el GRUN en procura de fortalecer los valores para que convivamos en un ambiente armonioso, con respeto al derecho ajeno, con concienciación respecto a los deberes ciudadanos y adoptando estilos de vida sanos. Con el fin primordial de erradicar la violencia en sus múltiples manifestaciones, en todos los ámbitos de la vida en sociedad y para alcanzar el anhelado desarrollo humano sostenible, lo cual solo es posible lograr a través de la cultura de paz promovida por nuestro buen gobierno.

\section{BIBLIOGRAFÍA Y REFERENCIAS}

19digital. (2018). Comandante Daniel Ortega: La lucha por la paz, sigue siendo un reto y un objetivo a alcanzar en el mundo. 19 digital. Obtenido de https://www.el19digital.com/articulos/ver/ titulo:85802-comandante-daniel-ortega-la-lucha-por-la-paz-sigue-siendo-un-reto-y-unobjetivo-a-alcanzar-en-el-mundo

19digital. (2019). 5 proyectos del 2019 marcados en la historia de Nicaragua. El 19 digital. Obtenido de https://www.el19digital.com/articulos/ver/titulo:97781-5-proyectos-del-2019-marcados-en-lahistoria-de-nicaragua-

19digital. (2019). Asamblea Nacional aprueba reforma a ley 977 para fortalecer lucha contra el terrorismo, crimen organizado y lavado de dinero. 19digital. Obtenido de https://www.el19digital.com/ articulos/ver/titulo:92868-asamblea-nacional-aprueba-reforma-a-ley-977-para-fortalecer-luchacontra-el-terrorismo-crimen-organizado-y-lavado-de-dinero

19digital. (2019). Ministra de Educación destaca logros en el cumplimiento de las metas de los Objetivos de Desarrollo Sostenible. 19digital. Obtenido de https://www.el19digital.com/articulos/ver/ titulo:95524-ministra-de-educacion-destaca-logros-en-el-cumplimiento-de-las-metas-de-losobjetivos-de-desarrollo-sostenible

19digital. (2019). Nicaragua sobrepasa la meta de electrificación para el 2019. Obtenido de https://www. el19digital.com/articulos/ver/titulo:98175-nicaragua-sobrepasa-la-meta-de-electrificacion-parael-2019

19digital. (2020). ¡Un sueño hecho realidad! Entregan primeras cien viviendas del programa Bismarck Martínez. 19 digital. Obtenido de https://www.el19digital.com/articulos/ver/titulo:101327-unsueno-hecho-realidad-entregan-primeras-cien-viviendas-del-programa-bismarck-martinez 
19digital. (2020). Gobierno Sandinista ha construido 18 hospitales desde el 2007. 19digital. Obtenido de https://www.el19digital.com/articulos/ver/titulo:101513-gobierno-sandinista-ha-construido-18hospitales-desde-el-2007

Asamblea, N. (1987). Constitución Política de la República de Nicaragua. Managua, Nicaragua: La Gaceta Diario Oficial. Obtenido de https://www.poderjudicial.gob.ni/pjupload/archivos/documentos/LA_ CONSTITUCION_POLITICA_Y_SUS_REFORMAS(3).pdf

Asamblea, N. (2019). Ley para una cultura de diálogo, reconciliación, seguridad, trabajo y paz (Vol. 17). Managua: La Gaceta, Diario Oficial. Obtenido de LEY PARA UNA CULTURA DE DIÁLOGO, RECONCILIACIÓN, SEGURIDAD, TRABAJO Y PAZ

GRUN. (2009). Plan nacional de desarrollo humano actualizado 2009-2011. Managua, Nicaragua. Obtenido de https://www.preventionweb.net/files/15491_pndh20092011.pdf

GRUN. (2017). Ejes del programa nacional de desarrollo humano. Managua, Nicaragua: Consejo de comunicación y ciudadanía. Obtenido de https://www.el19digital.com/app/webroot/tinymce/ source/2018/00-Enero/Del22al28Enero/Viernes26Enero/EJES\%20DEL\%20PROGRAMA\%20 NACIONAL\%20DE\%20DESARROLLO\%20HUMANO.pdf

MINREX. (2020). Mensaje del gobierno de reconciliación y unidad nacional al cuerpo diplomático acreditado en Nicaragua en el año nuevo 2020. págs. 5-6. Obtenido de https://www.el19digital.com/ app/webroot/tinymce/source/2020/00-Enero/06Ene/PALABRAS\%20DEL\%20CANCILLER\%20 DENIS\%20MONCADA-\%206\%20ENERO\%202020.pdf

PRONicaragua. (2019). Políticas y Proyectos de Desarrollo Para Potenciar la Inversión 2019-2020. Managua: PRO Nicaragua. Obtenido de WWW.PRONicaragua.gob.ni.

Visión, S. (2019). Frases emblemáticas del Comandante Carlos Fonseca. VIsiónsandinista. Obtenido de VIsiónsandinista 